\title{
Off-surface optic axis birefringent filters for smooth tuning of broadband lasers
}

\author{
UMIT DEMIRBAS ${ }^{1,2}$ * \\ ${ }^{1}$ Laser Technology Laboratory, Department of Electrical and Electronics Engineering, Antalya Bilim University, 07190 Antalya, Turkey \\ ${ }^{2}$ Center for Free-Electron Laser Science, The Hamburg Center of Ultrafast Imaging, Deutsches Elektronen Synchroton, Hamburg, Germany \\ *Corresponding author: umit79@alum.mit.edu
}

Received XX Month XXXX; revised XX Month, XXXX; accepted XX Month XXXX; posted XX Month XXXX (Doc. ID XXXXX); published XX Month XXXX

Transition metal-doped gain media such as Ce:LiCAF, Ti:Sapphire, Cr:ZnSe and Fe:ZnSe possesses wide gain bandwidths that could provide ultra broadly tunable laser output with the usage of adequate intracavity tuning elements. Birefringent filters (BRFs) are a low-cost and easy to use solution for tuning. However, for ultrabroad gain media, regular on-surface optic axis BRFs could not provide smooth tuning of laser wavelength in the whole emission range. Basically, regular BRFs could not accommodate a large enough free spectral range with acceptable modulation depth variation while tuning due to their slow tuning rates. Motivated by this, in this study we have numerically investigated the effect of optic axis orientation on filter parameters for magnesium fluoride birefringent tuning plates. We have shown that, a magnesium fluoride BRF with an optic axis diving by $30^{\circ}$ into the plate could provide smooth tuning of ultra-broad laser gain media. A similar analysis has shown that for broadband tuning applications the optimum optic axis diving angle lies around $25^{\circ}$ for crystal quartz BRFs. The proposed filters have the potential to be useful in tuning of broadband lasers in continuous-wave, long-pulsed and femtosecond operation regimes. (C) 2017 Optical Society of America

OCIS codes: 230.7408: Wavelength filtering devices; 140.3600: Lasers, tunable; 140.3580: Lasers, solid-state.

http://dx.doi.org/10.1364/AO.99.099999

\section{INTRODUCTION}

Birefringent tuning plates (birefringent filters: BRFs) are routinely employed in laser resonators for tuning of the output wavelength and/or for narrowing down the laser emission spectrum [1-3]. Since they can be inserted at Brewster's angle inside the laser cavity, they do not require anti-reflective coatings, and their passive losses are very low. This reduces their cost, enables broadband operation, and increases their damage threshold.

Important parameters to consider while optimizing the usage of birefringent tuning plates are free-spectral range (FSR), transmission passband bandwidth (FWHM, full-width at half-maximum), modulation-depth and tuning rate[4]. For optimization of filter parameters for a specific application, designers first usually adjust the thickness of the plate. Moreover, when one plate is not sufficient to reach the desired performance pairs of birefringent plates with different thicknesses could also be used.

Another less known knob that can be used to optimize the properties of birefringent filters is the angle between the optic axis and plates surface normal $[1,5-8]$. In standard usage, the plates optic axis lies on the surface of the plate for convenience in filter fabrication (can be named as on-surface optic axis birefringent filters or as regular birefringent filters). On the other hand, as several earlier studies with quartz birefringent filters has already shown, this particular case is not really optimum for many applications. Compared to regular birefringent filters, BRFs with optic axis pointing out of its surface provides a much broader set of filter parameters (named as diving optic axis birefringent filters or off-surface optic axis birefringent filters in the literature) $[1,3,6,9]$. In particular, wisely designed off-surface optic axis birefringent plates could: (i) generate larger FSR values, (ii) provide a smoother variation of modulation depth as the wavelength is tuned, (iii) enable faster tuning rates, and (iv) posses a larger range of usable filter rotation angles. For example, in the case of wavelength swept lasers , off-surface optic axis BRFs could provide faster tuning rates. Moreover, for some other applications, such as tuning of ultrabroad lasers (focus of this paper), it is not even possible to achieve the desired tuning properties with regular BRFs, and off-surface BRFs are the only solution (e.g., later see Fig. 11).

Unfortunately, the advantages of off-surface optic axis BRFs are not well known by our community, limiting researchers ability to optimize filters properties for their application. On top of this, as also pointed out by Kobtsev et al. [1], some of the earlier papers on off-surface optic axis birefringent filter design contain some errors, and optimal performance of these elements has not been fully described in the literature (in some cases not even correctly understood). Lastly, these earlier work mostly focused on optimization of birefringent filter properties for dye lasers which are relatively narrowband and to our knowledge there is limited study on optimization of BRF properties for 
today's broadly tunable lasers such as Ce:LiCAF $[10,11]$, Ti:Sapphire [12], Cr:ZnSe [13, 14], and Fe:ZnSe [15].

Motivated by this, in this study we present detailed numerical design considerations for off-surface optic axis magnesium fluoride birefringent tuning plates that are suitable for ultra broad tuning. As the birefringent material we have chosen to look at magnesium fluoride since it posses a much broader transparency range than the usually employed crystal quartz enabling its usage for the newly emerged near to mid-infrared laser materials (transparency range for quartz: $250 \mathrm{~nm}$ to $2500 \mathrm{~nm}$, transparency range for magnesium fluoride: $110 \mathrm{~nm}$ to $7500 \mathrm{~nm}$ ) [16]. In our analysis, we have looked at the effect of optic axis orientation and filter thickness on filter parameters such as free-spectral range, modulation depth, tuning-rate, and walk-off angle. We have shown that, for tuning of ultra-broad lasers, magnesium fluoride birefringent filters with an optic axis making $30 \pm 2^{\circ}$ with the plates surface normal provides a good solution. The optimum filter thickness value for this purpose has found to be $0.85 \mathrm{~mm}$ for Ce:LiCAF, $2.2 \mathrm{~mm}$ for Ti:Sapphire, $3.2 \mathrm{~mm}$ for Cr:Forsterite, $6 \mathrm{~mm}$ for Cr:ZnSe, and $11.5 \mathrm{~mm}$ for Fe:ZnSe. We have also looked at crystal quartz, and we have shown that quartz BRFs with an optic axis making $25 \pm 2^{\circ}$ with the plates surface normal could also provide smooth and ultra-broad tuning performance.

The paper is organized as follows. Section 2 summarizes the theory behind usage of intracavity birefringent filters as tuning elements in standing wave optical cavities. Section 3 presents detailed simulation results on the effect of optic axis orientation on filter performance. Section 4 describes thickness optimization process for a few selected laser gain media. Finally, Section 5 presents a summary of key findings and gives a generalized description for filter parameter optimization for wavelength tuning of other broadband laser gain media.

\section{THEORY}

Fig. 1 (a) shows a typical standing-wave laser cavity that includes a magnesium fluoride birefringent filter (BRF) and a broadband laser gain medium. Both the gain medium and the birefringent plate are inserted at Brewster's angle; since, otherwise they will both require broadband antireflective coatings, which increases complexity and cost. Tuning of the laser wavelength is facilitated simply by rotation of the birefringent plate about an axis normal to the surface (corresponds to changing $\rho$, in Fig. 1 (b)). The surfaces of the laser crystal and the birefringent plate will create Fresnel reflection losses for the transverse electric (TE/s) polarized part of the beam. Fig. 1 (b) shows a detailed view of the BRF, where $t$ is the thickness of the plate, $i$ is the incidence angle, $\beta$ is the internal Brewster's angle (around $36.1^{\circ}$ in magnesium fluoride at $2.5 \mu \mathrm{m}$ ), $\vec{s}$ is the direction of beam propagation in the plate, $\rho$ is the rotation angle of the plate, $\gamma$ is the angle between the crystals axis and the beam propagation direction and $\sigma$ is the angle between the optic axis and the surface normal (when $\sigma=90^{\circ}$, the optic axis lies on the surface of the plate, which is the typical BRF).

To calculate the transmission characteristic of the laser system [shown in Fig. 1 (a)] one needs to find the polarization eigenmodes and eigenvalues of the overall Jones matrix of the cavity. This problem has been studies in detail earlier and we will just provide a review of the results here for the sake of completeness [2, 4-6, 17-19]. We know form a physical point of view that, the polarization state should not change after a round-trip over the laser cavity. This involves solution of the following polarization eigenmode equation [5]:

$$
M_{\text {cavity }} \cdot \vec{E}=\varepsilon \vec{E},
$$

where $\vec{E}$ is the electric field vector (polarization eigenvector):

$$
\vec{E}=E_{T M} \hat{e}_{T M}+E_{T E} \hat{e}_{T E}
$$

$\mathrm{E}_{\mathrm{TE}}$ and $\mathrm{E}_{\mathrm{TM}}$ are the transverse-electric (TE/s) and transverse-magnetic $(\mathrm{TM} / \mathrm{p})$ polarized electric field vector components, and $\varepsilon$ is the polarization eigenvalue, which usually is a complex number. The eigenvalues $\left(\varepsilon_{1}\right.$ and $\left.\varepsilon_{2}\right)$ of the $2 \times 2$ round-trip Jones matrix for the laser cavity gives amplitude transmission coefficients for each polarization modes of the cavity, and $\left|\operatorname{Max}\left(\varepsilon_{1}, \varepsilon_{2}\right)\right|^{2}$ gives the power transmission for the relevant cavity polarization mode with the lowest loss [5].



Fig. 1. (a) Standing-wave laser resonator containing a gain medium and a birefringent plate inserted at Brewster's angle. (b) Light beam incident on the birefringent plate at Brewster's angle [2]. $\sigma$ : angle between the optic axis and the surface normal, $\vec{c}$ : optic axis, $\vec{s}$ : direction of beam propagation, i: incidence angle, $\beta$ : internal Brewster's angle, $\rho$ : rotation angle of the plate, $t$ : thickness of the plate.

The overall Jones matrix for a round-trip in our standing wave cavity can be written as:

$$
M_{\text {cavity }}=M_{\text {gain }} \cdot M_{B R F} \cdot M_{\text {gain }} \cdot M_{B R F},
$$

where Mgain and $\mathrm{MBRF}_{\mathrm{BR}}$ are the Jones matrixes for the gain medium and the birefringent plate, respectively. Note that the matrix above is for a standing wave cavity, where we pass through each element twice. For a ring cavity, the overall Jones matrix for the cavity will involve only one pass through each element $\left(\mathrm{M}_{\text {ring cavity }}=\mathrm{M}_{\text {gain }} \cdot \mathrm{M}_{\mathrm{brf}}\right)$. Also, when more than one BRF is used, the overall Jones matrix should include the Jones matrices for all the birefringent filters. The Jones matrix for the gain medium (which acts as a partial polarizer) can be written as[5]:

$$
M_{\text {gain }}=\left(\begin{array}{cc}
1 & 0 \\
0 & q_{\text {gain }}^{2}
\end{array}\right),
$$

where $q_{\text {gain }}=2 n /\left(n^{2}+1\right)$ is the transmission coefficient of the TE polarized electric field at Brewster's angle for the gain medium, and $n$ is the refractive index (e.g., for Cr:ZnSe, refractive index around $2.5 \mu \mathrm{m}$ is 2.44 , Brewster's angle is $67.7^{\circ}$, and $\mathrm{q}_{\mathrm{znse}}=0.71$ ). For the Brewster's angle inserted BRF the Jones matrix can be written as [2,5]:

$$
M_{B R F}=\left(\begin{array}{cc}
\operatorname{Cos}^{2}(\theta)+\operatorname{Sin}^{2}(\theta) \operatorname{Exp}(j \Delta \phi) & q_{b f f} \operatorname{Cos}(\theta) \operatorname{Sin}(\theta)[\operatorname{Exp}(j \Delta \phi)-1] \\
q_{b f f} \operatorname{Cos}(\theta) \operatorname{Sin}(\theta)[\operatorname{Exp}(j \Delta \phi)-1] & q_{b f f}^{2} \operatorname{Cos}^{2}(\theta)+\operatorname{Sin}^{2}(\theta) \operatorname{Exp}(j \Delta \phi)
\end{array}\right)
$$

In (5), q $\mathrm{brf}_{\mathrm{b}}$ is the transmission coefficient of the TE polarized electric field at Brewster's angle (fore magnesium fluoride, refractive index is around 1.37 , Brewster's angle is $\left.53.9^{\circ}, \mathrm{q}_{\mathrm{br}}=0.916\right), \Delta \phi$ is the phase retardation of the plate and can be calculated using:

$$
\Delta \phi=\frac{2 \pi}{\lambda} \frac{t}{\operatorname{Cos}(\beta)}\left(n_{e}-n_{o}\right) \operatorname{Sin}^{2}(\gamma)=\frac{2 \pi}{\lambda} \frac{t \Delta n}{\operatorname{Cos}(\beta)} \operatorname{Sin}^{2}(\gamma) .
$$


In Eq. (6), $n_{o}$ and $n_{e}$ are the ordinary and extraordinary indexes of refraction for the BRF material, and for magnesium fluoride a Sell Meier type dispersion equation given in Table I of [16] has been used for calculation of the wavelength dependence of birefringence $\Delta n=\left(n_{e^{-}}\right.$ $\mathrm{n}_{0}$ ). The angles $\theta$ and $\gamma$ appearing in equations (5) and (6) can be calculated in terms of the other more accessible angles using:

$$
\begin{gathered}
\operatorname{Cos}(\gamma)=\operatorname{Cos}(\beta) \operatorname{Cos}(\sigma)+\operatorname{Sin}(\beta) \operatorname{Sin}(\sigma) \operatorname{Cos}(\rho), \\
\operatorname{Cos}(\theta)=-\frac{\operatorname{Sin}(\sigma) \operatorname{Sin}(\rho)}{\operatorname{Sin}(\gamma)} .
\end{gathered}
$$

Figure 2 depicts the situation using an index ellipsoid for better visibility. Note that $\gamma$ is the angle between the optic axis of the birefringent crystal and the beam propagation direction (angle between $\vec{c}$ and $\vec{s}$ as shown in Fig. 1 (b) also), and $\theta$ is the angle between the TM polarized part of the electric field ( incident light beam and the ordinary refractive axis of the birefringent plate $\left(\hat{e}_{o}\right)$. We note here that there is a mistake in the stated equation for the calculation of $\theta$ in [2], and the correct from is given in Eq. (8).



Fig. 2. Index ellipsoid of the magnesium fluoride crystal that posses a positive uniaxial birefringence [2]. Projection of the incident electric field onto the crystallographic axis of the filter has also shown. $\mathrm{n}_{\mathrm{o}}$ : ordinary refractive index, ne: extraordinary refractive index, $n_{e}(\gamma)$ : refractive index observed by the extraordinary wave in $\mathrm{kDB}$ formalism, $\vec{c}$ : optic axis, $\vec{s}$ : direction of beam propagation, $\gamma$ : angle between $\vec{c}$ and $\vec{s}$.

While using the birefringent filter inside the laser resonator, the laser wavelengths that satisfy:

$$
\Delta \phi=m 2 \pi
$$

relation will not be effected by the birefringent filter (will see it as a fullwave plate). For our specific cavity described, then the solution of Eq. (1) shows that, at these wavelengths the filter transmission is unity and the polarization eigenmode has only the TM component. The wavelengths at which Eq. (9) holds can be calculated using [5]:

$$
\lambda_{m}=\frac{t \Delta n \operatorname{Sin}^{2}(\gamma)}{m \operatorname{Cos}(\beta)} .
$$

When the wavelength is $\lambda_{\mathrm{m}}$ ( $\mathrm{m}^{\text {th }}$ resonance wavelength), the polarization is TM at all the interfaces, and the beam will not observe any loss. On the other hand, other wavelengths on both sides of $\lambda_{m}$ will have elliptic polarization and will observe loss due to their TE component of the electric field.
Tuning of the laser wavelength is facilitated by rotation of the plate about an axis normal to the surface (corresponds to changing $\rho$, which will change $\gamma$ and hence the peak transmission wavelength $\lambda_{\mathrm{m}}$ ). Hence the tuning rate could be expressed as $\left(\mathrm{d} \lambda_{\mathrm{m}} / \mathrm{d} \rho\right)$ :

$$
\text { Tuning_Rate }=\frac{t \Delta n \operatorname{Sin}(2 \gamma)}{m \operatorname{Cos}(\beta)} \frac{\partial \gamma}{\partial \rho},
$$

and using Eq. (10) can be re-written as:

$$
\text { Tuning_Rate }=\frac{\lambda \operatorname{Sin}(2 \gamma)}{\operatorname{Sin}^{2}(2 \gamma)} \frac{\partial \gamma}{\partial \rho} .
$$

Achieving lasing only at a single wavelength at each instant during tuning requires the filter to have only one transmission maxima in the tuning range. In other words the filter free spectral range (FSR, spacing in optic wavelength between two successive transmission maxima) should be larger than gain bandwidth of the laser medium. One can calculate the free spectral range of the filter using [5]:

$$
F S R=\frac{\lambda^{2} \operatorname{Cos}(\beta)}{t \Delta n \operatorname{Sin}^{2}(\gamma)} .
$$

Note that, using Eq. (10), the equation for the FSR can also be rewritten as [5]:

$$
F S R=\frac{\lambda}{m}
$$

Another important property of the birefringent filter is the modulation depth. Basically, when the laser resonance is tuned to a specific wavelength, for the neighboring wavelengths the loss should be high enough to suppress lasing. The modulation depth (MD) of the filter can be calculated using [2]:

$$
M D=4 \operatorname{Cos}^{2}(\theta) \operatorname{Sin}^{2}(\theta) .
$$

The modulation depth could be maximized at $\theta$ values of $\pm 45^{\circ}$ and \pm 135 by exciting the ordinary and the extraordinary waves equally. Also $\theta=0^{\circ}$ and $\theta=90^{\circ}$, excites only the ordinary or extraordinary waves, and do not produce any modulation.

Lastly, as it is well-known, in birefringent crystals, incident ray is divided into an ordinary and extraordinary ray, and the walk-off angle (WOE, the angle between ordinary and the extraordinary rays) could be estimated using [6]:

$$
\begin{aligned}
& \text { WOE }=\frac{\Delta n}{n_{o}} \frac{\operatorname{Sin}(\gamma)}{\operatorname{Cos}(\beta)} \times \\
& \sqrt{[\operatorname{Sin}(\gamma) \operatorname{Sin}(\beta) \operatorname{Sin}(\eta)]^{2}+[2 \operatorname{Cos}(\gamma)+\operatorname{Sin}(\beta) \operatorname{Sin}(\gamma) \operatorname{Cos}(\eta)]^{2}}
\end{aligned}
$$

where

$$
\operatorname{Cos}(\eta)=\frac{\operatorname{Cos}(\beta) \operatorname{Sin}(\sigma) \operatorname{Cos}(\rho)-\operatorname{Sin}(\beta) \operatorname{Cos}(\sigma)}{\operatorname{Sin}(\gamma)}
$$

Note than in the derivations for filter properties, weak birefringence is assumed, $\Delta n / n_{0}<<n_{0}\left(\Delta n / n_{0}\right.$ is less than $1 \%$ for magnesium fluoride), but for the estimation of walk-off angle the first order effect is included [6]. Lastly, the separation (D) between the ordinary and the extraordinary rays at the exit face of the BRF could be estimated using:

$$
D=\frac{t}{\operatorname{Cos}(\beta)} \operatorname{Tan}(W O E) \text {. }
$$

\section{DESIGN DISCUSSION}

The aim of this design effort is to calculate the optimum cut angle $(\sigma)$ and optimum thickness $(\mathrm{t}$ ) for a magnesium fluoride birefringent tuning plate that could smoothly tune broad-bandwidth laser gain media such as Ti:Sapphire, Cr:ZnSe, and Fe:ZnSe. In our discussion 
below, we will try to speak in general terms. However, as a specific example, we will work on Cr:ZnSe, which has a gain spectrum covering the region roughly from $\lambda_{1}=1.8 \mu \mathrm{m}$ to $\lambda_{2}=3.4 \mu \mathrm{m}[13,14]$. A freespectral range of around $1.6 \mu \mathrm{m}$ is required for tuning $\mathrm{Cr}: \mathrm{ZnSe}$ around the central wavelength of $2.35 \mu \mathrm{m}$ (harmonic mean). Mathematica has been used in all the calculations that will be presented below.

\section{A. Effect of Optic Axis Direction on Free Spectral Range}

Since we are interested with ultrabroad tuning of solid-state lasers and hence large free spectral ranges (FSRs), we start our discussion by looking at the effect of birefringent filter's crystal axis orientation $(\sigma)$ on FSR. Figure 3 shows the calculated variation of filter FSR with filter rotation angle $\rho$. The calculation has been performed at a central wavelength of $2.35 \mu \mathrm{m}$, for several different optic axis orientations $\sigma$ in the range from $0^{\circ}$ and $90^{\circ}$, for a magnesium fluoride birefringent tuning plate with a thickness of $3 \mathrm{~mm}$. Here, we take the thickness as 3 $\mathrm{mm}$ as a reasonable value which will enable us to start a discussion, and optimization of the filter thickness will be discussed in Section 2.E.

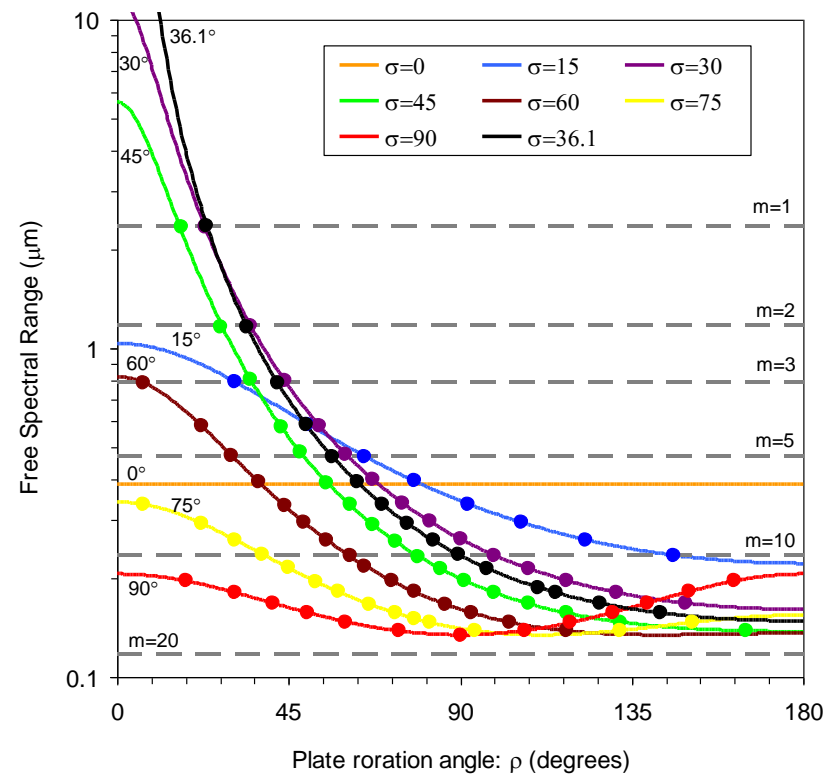

Fig. 3. Calculated variation of free spectral range for a standing-wave Cr:ZnSe laser cavity around $2.35 \mu \mathrm{m}$, as a function of birefringent plate rotation angle $(\rho)$. The calculation has been performed for different optic axis orientation values $\sigma$ ranging between $0^{\circ}$ and $90^{\circ}$. The magnesium fluoride birefringent plate was assumed to have a thickness of $3 \mathrm{~mm}$.

Note that in Fig. 3, the $x$-axis has been limited to plate rotation angle values from $\rho=0^{\circ}$ to $\rho=180^{\circ}$, and the $180^{\circ}-360^{\circ}$ range is a symmetric copy. The solid lines have been calculated using Eq. (13), and the dots on the solid lines are the resonance points. These are the points where Eq. (10) is satisfied, and the plate produces a phase shift of $m$ times $2 \pi$. Also, the dashed gray horizontal lines in the figure indicates the $\lambda / \mathrm{m}$ values, at selected $m$ values of 1, 2, 3, 5, 10 and 20 (Eq. (14)). Note that, even though Eq. (13) predicts a continuous set of FSR values, in reality at a central wavelength of $\lambda$, we can only achieve quantized FSR values of $\lambda / \mathrm{m}$. Hence, the largest FSR value that can be achieved from a BRF at a central wavelength of $\lambda$ is $\lambda$ (for the case $m=1$ ), and calculated FSR values that are larger than the central wavelength do not have physical significance. Moreover, the thickness of the plate as well as its cut angle $(\sigma)$ determines which orders (which $m$ values) will be available for a specific filter.

We see clearly from Fig. 3 that, birefringent filters diving angle $(\sigma)$ significantly effects the range of FSR values that can be obtained. Basically FSR is proportional to $\operatorname{Sin}^{-2}(\gamma)$, and $\gamma$ is a function of $\sigma$, as well as $\rho$, so we are scanning a two dimensional surface (which enables better optimization of filter properties). First of all, when $\sigma=0^{\circ}$, the plate's crystal axis is perpendicular to the plate surface, $\theta=90^{\circ}$ and $\gamma=\beta$ (the internal Brewster's angle, $36.1^{\circ}$ ), and $\gamma$ is independent of $\rho$ (the incident beam only excites the extraordinary polarization). Hence, the $\mathrm{BRF}$ does not change the polarization state of the incident beam (which is in an eigen polarization direction), and the calculated FSR values do not have physical significance (transmission is independent of wavelength $(100 \%))$. Here we are assuming any other possible depolarization effect of the materials such as chirality is small.

When the plates optic axis lies on the surface of the plate $\left(\sigma=90^{\circ}\right.$, typical on-surface optic axis BRF), the obtainable FSR values are quite small and varies in a very narrow range $(130-200 \mathrm{~nm})$. This is because for this specific thickness $(3 \mathrm{~mm})$, at this specific central wavelength $(2.35 \mu \mathrm{m})$, when $\sigma=90^{\circ}$ the BRF enables accessing plate orders (m) between 12 and 17 only, and this limits the obtainable FSR values to between $\lambda / 12 \approx 196 \mathrm{~nm}$ and $\lambda / 17 \approx 138 \mathrm{~nm}$.

This is further illustrated in Fig. 4, where we plot variation of filter resonance wavelength as a function of rotation angle $(\rho)$, for plate optic axis orientation $(\sigma)$ values of $30^{\circ}$ and $90^{\circ}$. The horizontal gray line in Fig. 4 indicates the central wavelength for Cr:ZnSe $(2.35 \mu \mathrm{m})$. Note that, the rate of change of the resonance wavelength with rotation angle $\rho$ is the tuning rate (Eq. 11) and for broadly tunable gain media a large tuning range is generally desired. Therefore, the slope of the curves in Fig. 4 gives the tuning rate of the filter for the specific order (the steeper the better). The effect of plate optic axis orientation $(\sigma)$ on tuning rate will be discussed in more detail in the next section.



Fig. 4. Calculated variation of filter resonance wavelength as a function of birefringent plate rotation angle $(\rho)$. The calculation has been performed for optic axis orientation values of $\sigma=30^{\circ}$ and $\sigma=90^{\circ}$ (the later has been shown with dashed lines). The magnesium fluoride birefringent plate was assumed to have a thickness of $3 \mathrm{~mm}$.

For the specific case of Cr:ZnSe, we desire an FSR value of $1.6 \mu \mathrm{m}$ around $2.35 \mu \mathrm{m}$, which requires us to reach an $\mathrm{m}$ value of 1 (even $\mathrm{m}=2$ which provides an FSR of $1.175 \mu \mathrm{m}$ will not be sufficient). As it is clear from Fig. 4 also, a regular BRF with an on-surface optic axis $\left(\sigma=90^{\circ}\right)$, only has orders between $m=12$ and $m=17$ for $3 \mathrm{~mm}$ thickness, and is not an option. By decreasing the filters thickness from $3 \mathrm{~mm}$ to $0.2 \mathrm{~mm}$, it is possible to achieve an order of $m=1$ for an on-surface optic axis BRF also. However, such filters also posses very slow tuning rates, limiting their usage in tuning (will be discussed later, e.g., Fig. 11).

We see from Fig. 3 that, very large values of FSR could be obtained for small plate rotation angels $\left(\rho: 0-45^{\circ}\right)$, when the optic axis orientations $\sigma$ is in the $25-45^{\circ}$ range.Especially, as $\sigma$ gets closer to $\beta$ (the internal Brewster's angle, $36.1^{\circ}$ ), things get quite interesting. At 
this angle $(\sigma=\beta)$, for small $\rho$ values, the direction of beam propagation $\vec{s}$ gets closer to the direction of crystals c axis $(\vec{c})$, and $\gamma$ approaches an angle of $0^{\circ}$. Since the FSR scales inversely with $\operatorname{Sin}^{2}(\gamma)$, as $\gamma$ approaches $0^{\circ}$, the calculated FSR values increase sharply, and diverges to infinity at $\sigma=\beta$. However we should note here that, when this happens $\left(\gamma \rightarrow 0^{\circ}\right)$, the phase retardation of the plate $(\Delta \phi)$ also approaches zero (Eq. (6)) indicating that, the obtained FSR values does not have any physical significance (there is no transmission maxima for $2.35 \mu \mathrm{m}$ ). One can also see this in Fig. 2, when $\gamma=0$, $n_{e}(\gamma)=n_{o}$, and there is no phase difference between the ordinary and the extraordinary waves.

To summarize, from Fig. $3 \& 4$, we see that optic axis orientations $(\sigma)$ especially in the $20-45^{\circ}$ range is interesting, since for a given birefringent filter thickness, they enable resonance for a larger set of $\mathrm{m}$ values. For example, when $\sigma=30^{\circ}$, the BRF plate provide resonances for $m$ values between 1 and 14, enabling FSR values between $\lambda / 1 \approx 2.35$ $\mu \mathrm{m}$ and $\lambda / 14 \approx 168 \mathrm{~nm}$. For our design aim, reaching $\mathrm{m}=1$ is important in attaining the largest possible FSR value. On a more general perspective, a BRF that could provide a large variety of filter orders is capable of producing a rich set of filter parameters. This is in general very useful, since it enables optimization of performance for different applications. As an example, a recent study demonstrated advantages of off-surface optic axis BRFs in multicolor lasing $[9,20]$.

\section{B. Effect of Optic Axis Direction on Tuning-Rate}

In our discussion so far, we have focused our attention on optimization of free-spectral range. As mentioned above, another important parameter for a birefringent filter is its tuning rate. As we will see in Section 3.D, as we rotate the filter (by varying $\rho$ ), the modulation depth of the filter also changes. If the designed filter is not tuning fast enough, then as one tunes the wavelength, this could result in significant changes in modulation depth, and might result in undesired effects such as wavelength jumps, etc... Hence, for our application here, in tuning ultra-broad laser gain media, we require tuning rates roughly in the order of FSR/10 per degree of plate rotation. Then, for the Cr:ZnSe laser with a central wavelength of $2.35 \mu \mathrm{m}$ and a desired FSR of $1.6 \mu \mathrm{m}$, a tuning rate in the order of $100-200 \mathrm{~nm} /$ degree is desired.

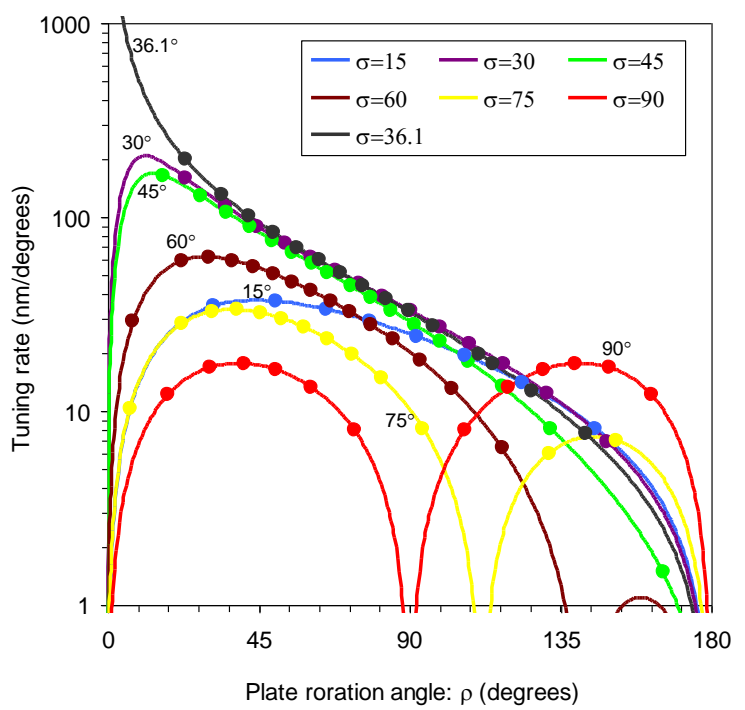

Fig. 5. Calculated variation of tuning rate for a standing-wave Cr:ZnSe laser cavity around $2.35 \mu \mathrm{m}$, as a function of birefringent plate rotation angle $(\rho)$. The calculation has been performed for different optic axis orientation values $\sigma$ ranging between $0^{\circ}$ and $90^{\circ}$. The results are independent of filter thickness.

To investigate this issue, Fig. 5 shows the calculated variation of filter tuning rate as a function of filter rotation angle $\rho$ (using Eq. 12, the calculation is independent of filter thickness). Note that, similar to the FSR, acquiring large tuning rates require usage of the filter at small rotation angles $\left(0-45^{\circ}\right.$ range). Moreover, the regular on-surface optic axis birefringent filter $\left(\sigma=90^{\circ}\right)$ provide very small tuning rates, and again is not a good choice. On the other hand, off-surface optic axis BRFs with a diving angle $(\sigma)$ between $25-45^{\circ}$ enables large tuning rates up to $150-200 \mathrm{~nm} /$ degree, indicating that the full tuning-range (1.8-3.4 $\mu \mathrm{m}$ ) could be scanned by rotating the plate (changing $\rho$ ) $8-12^{\circ}$ only. This finding will be important in our discussion of modulation depth in the next section.

\section{Effect of Optic Axis Direction on Modulation Depth}

In the earlier sections, we have seen that filters with $\sigma$ between $25-45^{\circ}$ are good candidates for broadband tuning, when used in rotation angles between $0-45^{\circ}(\mathrm{m}=1)$. However, for the $\mathrm{BRF}$ to work properly it should also provide enough modulation depth to suppress neighboring side wavelengths so that clean single wavelength operation is achieved (might especially become an issue when the gain is high and output coupling and other cavity losses are low). For that, first of all we desire a modulation depth as large as possible. Moreover, as we tune the wavelength, we want to keep the modulation depth as smooth as possible to obtain the same filter rejection across the whole tuning range (we need to rotate the filter around $10^{\circ}$ to cover the full tuning range, and the modulation depth should not change a lot as we tune).
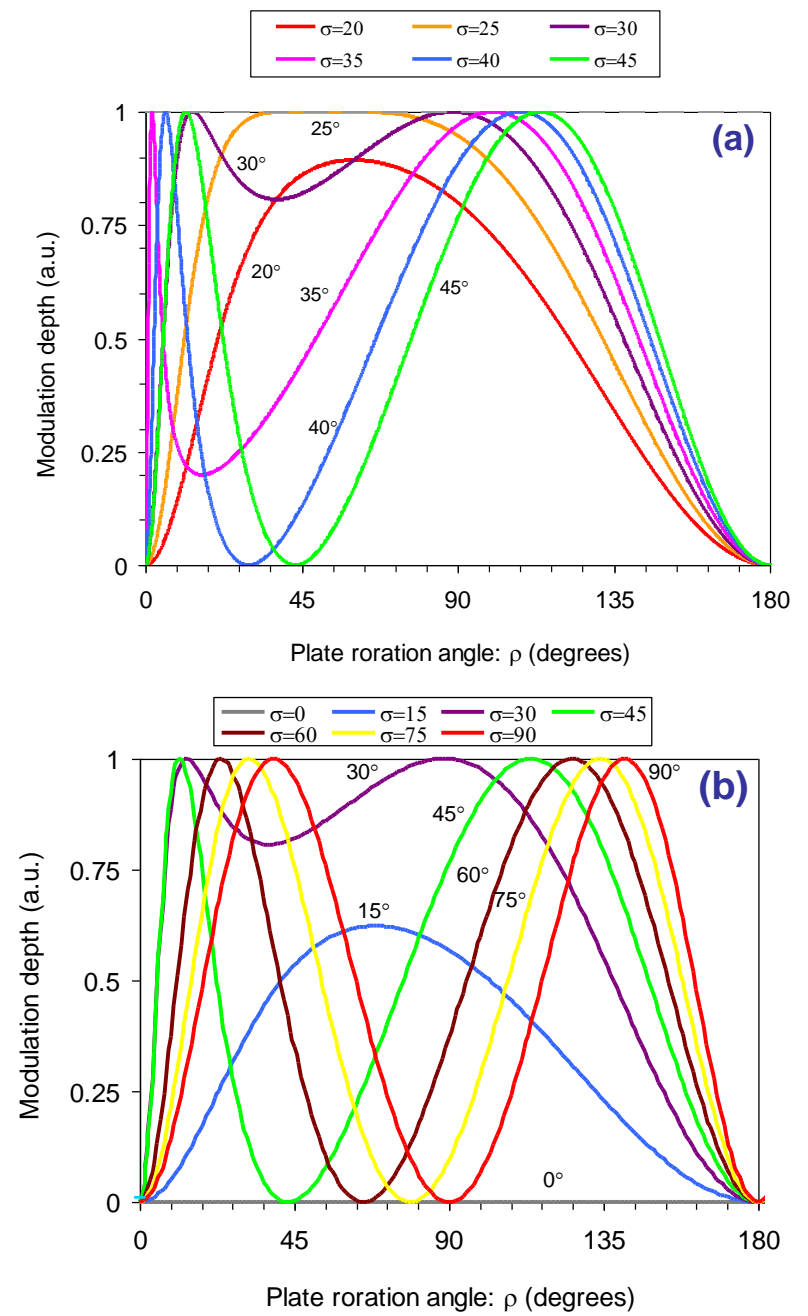

Fig. 6. Calculated variation of modulation depth as a function of magnesium fluoride birefringent plate rotation angle ( $\rho)$. The calculation has been performed for different optic axis orientation values $\sigma$ ranging between $0^{\circ}$ and $90^{\circ}$ (see differently colored graphs). 
The graph is divided into two for easier visibility. The results are independent of filter thickness.

To explore this, Fig. 6 shows the calculated variation of filter modulation depth as a function of filter rotation angle. What is important for our discussion is the modulation depth behavior of the filter for optical axis orientation $(\sigma)$ values in the $20^{\circ}-45^{\circ}$ range (Fig. 6 (a) presents curves around this region). We see that $\sigma$ values that are very close to $\sigma=\beta=36.1^{\circ}$, such as $\sigma=35^{\circ}$ is not very useful, since they provide a very narrow region of operation in the desired rotation angle range $\left(\rho: 0-45^{\circ}\right)$. Basically, modulation depth is high in a very narrow range around $10^{\circ}$ of rotation angle, then drops sharply. Same is true for $\sigma=40^{\circ}$ and $\sigma=45^{\circ}$, they also provide a very narrow operation region, where as we need a smooth modulation depth for at least $10^{\circ}$ of rotation. On the other hand, $\sigma=25^{\circ}$ provides a very broad tuning angle range where the modulation depth is almost constant (the smoothest curve is obtained at $\sigma=24.6^{\circ}$, and this angle can be calculated using $\sigma_{\text {flat }}=\operatorname{ArcCos}\left(1 / \sqrt{2\left(1+n^{2}\right)}\right)$ [1]). However, for $\sigma=25^{\circ}$, the useful tuning angle range for $\rho$ is shifted to rotation angles larger than $30^{\circ}$, where the FSR and tuning rate starts to decrease. In that respect, $\sigma=30^{\circ}$ provides a nice balance, where the modulation depth is relatively smooth and has a shoulder at smaller rotation angle values $\left(\rho=13.6^{\circ}\right)$, where large tuning rates could be attained.

We can numerically confirm our observations above by looking at Fig. 7 , which shows the calculated variation of average modulation depth as a function of birefringent plate optic axis diving angle $\sigma$. Average modulation depth has been calculated for two different intervals: (i) red solid line in the 0 to $\pi$ range, and (ii) green dashed line in the 0 to $\pi / 4$ range (e.g., curves like in Fig. 6 is integrated between $\rho$ values of 0 and $\pi / 4$, and then the integrant is divided by $\pi / 4$ to calculate an average value of modulation). Fig. 7 also shows the variation of optimum plate rotation angle as a function of plates optic axis diving angle $\sigma$. Here the solid blue curve shows the optimum rotation angle $\rho$ for maximizing the modulation depth, where as the dashed purple curve shows the optimum rotation angle $\rho$ for maximizing the tuning rate.



Fig. 7. Calculated variation of average modulation depth for a magnesium fluoride birefringent filter as a function of optic axis orientation $\sigma$. Variation of optimum plate rotation angle for tuning rate (TR) and modulation depth (MD) are also shown. Expected tuning performance of magnesium fluoride birefringent plates for ultra broad lasers is optimum at a cut angle of $\sigma=28.5^{\circ}$. The results are independent of filter thickness.

First of all, note from Fig. 7 that the average modulation depth one can get is maximized at an optic axis diving angle of $28.8^{\circ}$ and $28.5^{\circ}$ when averaged in $0^{\circ}-180^{\circ}$ and $0^{\circ}-45^{\circ}$ intervals, respectively. On the other hand, as we see these are not sharp optimums and cut angles around these values should also provide reasonable performance. Considering this, here we would like to propose a cut angle of $30 \pm 2^{\circ}$ for magnesium fluoride BRFs, as this might be easier to produce experimentally. As the cut angle tolerance, $\pm 2^{\circ}$ should be given, since especially for cut angles above $32^{\circ}$ (as $\sigma$ approaches $\beta$ ), there is a sharp decrease in filter performance in the region of interest.

Fig. 7 also plot the variation of optimum plate rotation angle $\rho$ for attaining the largest tuning rate and largest modulation depth as a function of optic axis diving angle. Basically, if the optimum rotation angle of the BRF for maximizing tuning rate (TR) and modulation depth (MD) is too much away from each other $\left(>15^{\circ}-20^{\circ}\right)$, then it will be harder to achieve the desired tuning performance. For example, looking at Fig. 7, we see than that, $\sigma$ values especially in the $10-25^{\circ}$ range is not suitable because of this issue. For $\sigma=30 \pm 2^{\circ}$, we have very close optimum rotation angle values for tuning rate and modulation depth maximization $\left(11.1^{\circ}\right.$ and $\left.13.6^{\circ}\right)$, confirming the suitability of setting $\sigma$ around $30^{\circ}$. In the following, we will assume an optimum cut angle of $\sigma=30 \pm 2^{\circ}$ and an optimum rotation angle $(\rho)$ of $13.6^{\circ}$ for the magnesium fluoride birefringent tuning plate.



Fig. 8. Calculated variation of average modulation depth for a quartz birefringent filter as a function of optic axis orientation value $\sigma$. Variation of optimum plate rotation angle for tuning rate (TR) and modulation depth (MD) are also shown. Expected tuning performance of quartz birefringent plates for ultra broad lasers is optimum at a cut angle of $\sigma=26.1^{\circ}$. The results are independent of filter thickness.

Lastly, we would like to present Fig. 8, where we have summarized the same optimization process for the well-known crystal quartz material (curve is now calculated at a central wavelength of $0.85 \mu \mathrm{m}$ ). First of all, the shape of the curves for quartz and magnesium fluoride looks quite similar (Figs 7 \& 8), as it is somehow expected, since both are positive uniaxial materials. There is a slight change in the optimum cut angle, and for our application for quartz birefringent plates the optimum is around $26.1-26.4^{\circ}$. Here we would like to propose a cut angle of $25 \pm 2^{\circ}$ for quartz, as again this might be an easier selection for production. Note that the corresponding optimum rotation angle of $\rho$ is around $23.5^{\circ}$. As a side note, the internal Brewster angle for quartz is ( $\left.\beta_{\text {quartz }}\right)$ is around $33^{\circ}$, which is smaller compared to magnesium fluoride $\left(\beta_{\mathrm{mg} 2} \approx 36^{\circ}\right)$. That is one of the reasons for not choosing a cut angle of $30^{\circ}$ for quartz, since small production errors and alignment issues might result in unexpected drop in filter performance as $\sigma$ approaches $\beta$.

\section{Effect of Optic Axis Direction on Walk-off Angle}

In this section, we would like to investigate the effect of filter rotation angle $\rho$ and filter optic axis orientation $\sigma$ on the walk-off angle. Figure 9 shows the calculated variation of walk-off angle as a function of these 
parameters. From Fig. 9, we see that for the proposed cut angle of $\sigma=30^{\circ}$, around the optimum rotation angle $\rho$ of $13.5^{\circ}$, the estimated walk-off angle is only around $0.15^{\circ}$. For a birefringent plate thickness of $10 \mathrm{~mm}$, the corresponding beam separation (D) at the output is then around $30 \mu \mathrm{m}$. Generally speaking, BRFs should be placed at places where the beam size on the filter is at least an order of magnitude larger than the expected walk-off (otherwise, this might pose practical problems especially in designs where the usage of thick birefringent filters are required). In general this is well-satisfied when the BRF is inserted inside the cavity near the flat high-reflector or the output coupler, where the beam sizes are relatively large. As an example, a $33 \mathrm{~mm}$ thick quartz BRF is used in [21] inside a Ti:Sapphire laser without any specific problem (quartz has similar walk-off [6]).

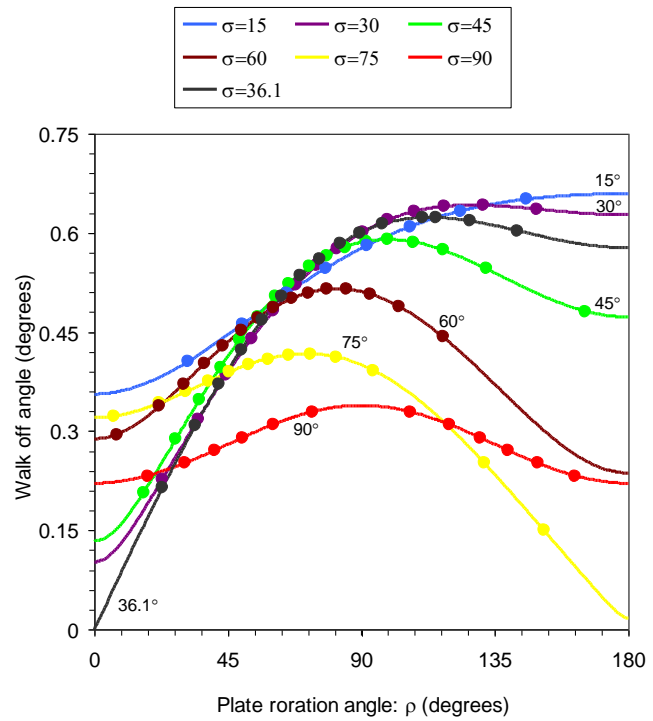

Fig. 9. Calculated variation of walk-off angle as a function of magnesium fluoride birefringent plate rotation angle $(\rho)$. The calculation has been performed for different optic axis orientation values $\sigma$ ranging between $15^{\circ}$ and $90^{\circ}$ (see differently colored graphs). The results are independent of filter thickness.

\section{E. Optimization of Filter Thickness}

In the earlier sections, we have shown that a plate cut angle of $30^{\circ}$ is a good choose for magnesium fluoride BRFs to obtain filters with large FSR values, large tuning rates and smooth modulation depth. Moreover, once $\sigma$ is taken as $30^{\circ}$, the plate should be used at around a rotational angle $(\rho)$ of $13.6^{\circ}$, to operate at the desired region $(\mathrm{m}=1$, with maximal FSR value and tuning rate). A similar argument applies to crystal quartz as well $\left(\sigma=25^{\circ}, \rho=23.5^{\circ}\right)$. Once, $\sigma$, $\rho$ and $m$ are chosen, and central operating wavelength $(\lambda)$ is known, the optimum thickness could be calculated from $\mathrm{Eq}(10)$ :

$$
t_{\text {opt }}=\frac{\lambda m \operatorname{Cos}(\beta)}{\Delta n \operatorname{Sin}^{2}(\gamma)} .
$$

\section{DESIGN EXAMPLES}

\section{A. Broadband Filter Design for Cr:ZnSe Lasers}

As mentioned above, Cr:ZnSe is a broadly tunable laser gain media in the near-infrared $(1880-3100 \mathrm{~nm})[13,14]$, with a gain peak around $2.35 \mu \mathrm{m}$. Using Eq. (10.a), we find the optimum thickness for the birefringent filter as $6.195 \mathrm{~mm}$ (can be roughly taken as $6 \mathrm{~mm}$ ). Figure 10 shows the calculated transmission characteristics of a standingwave Cr:ZnSe laser containing $3 \mathrm{~mm}$ (a), $6 \mathrm{~mm}$ (b) and $12 \mathrm{~mm}$ (c) thick magnesium fluoride BRFs with an optic axis diving angle of $30^{\circ}$. We see that for the $6 \mathrm{~mm}$ filter, as the filter angle is varied in the $10-19^{\circ}$ range, the transmission maxima of the filter shifts from $1.7 \mu \mathrm{m}$ to $3.4 \mu \mathrm{m}$, and the modulation depth stays in the $70-80 \%$ range as the filter is tuned, which shows that this filter could successfully accomplish smooth tuning of Cr:ZnSe laser in its whole emission range.
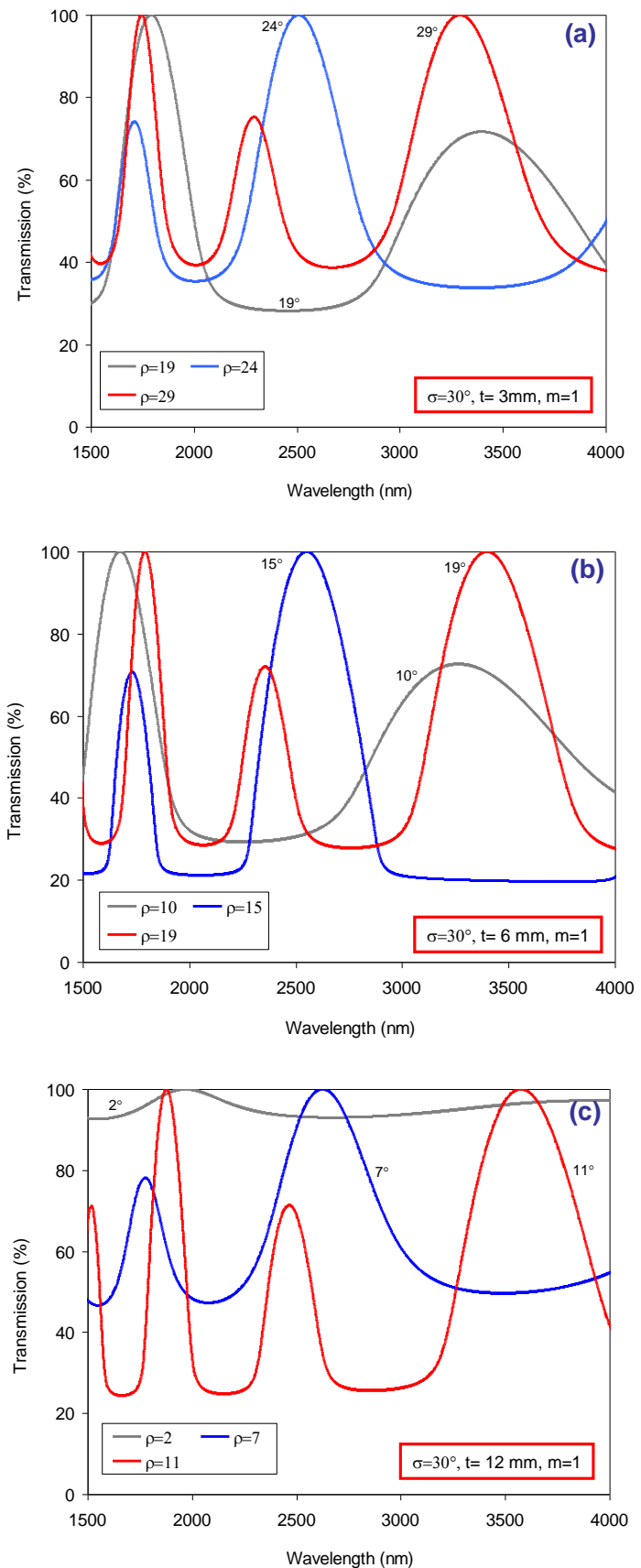

Fig. 10. Calculated tuning characteristics of a standing-wave Cr:ZnSe laser cavity using birefringent filter with thicknesses of (a) $3 \mathrm{~mm}$, (b) $6 \mathrm{~mm}$ and (c) $12 \mathrm{~mm}$. The calculation has been performed for a magnesium fluoride birefringent plate with an optic axis orientation $(\sigma)$ of $30^{\circ}$. Plate rotation angles are chosen so that the laser tunes around the first order $(\mathrm{m}=1)$, where the FSR is largest.

When the filter thickness $(\mathrm{t})$ is away from the optimum value $(6 \mathrm{~mm}$ for the case of $\mathrm{Cr}: \mathrm{ZnSe}$ ), to attain the same FSR value, or to operate near filter order $m=1$, one needs to use rotation angle values away from $13.6^{\circ}$. For example, a filter thickness of $3 \mathrm{~mm}$ requires operating around $23^{\circ}$, and a filter thickness of $12 \mathrm{~mm}$ needs operation near $6^{\circ}$. As we see from Fig. 10.c, for the $12 \mathrm{~mm}$ filter this will result in operating at a region with significantly lower modulation depth. On top of this, there is significant variation in modulation depth as the filter is 
tuned. Moreover, a thick filter will also suffer from larger beam walkoff and larger material dispersion, and hence is not preferable anyway. Similarly, a $3 \mathrm{~mm}$ thick filter, which needs to operate around $23^{\circ}$, faces larger variation in modulation depth as it is tuned. On the other hand, as we see from Fig. 10 (a), the filters performance for this case might still be acceptable for some applications, considering additional advantages in terms of cavity dispersion and walk-off angle minimization.

It is also interesting to look at the performance of a regular birefringent filter with an on-surface optic axis $\left(\sigma=90^{\circ}\right)$. Note that for this cut angle, modulation depth is optimized at a rotation angle $\rho$ of $40^{\circ}$, then to operate around the first resonance $(\mathrm{m}=1)$, one needs a filter with a thickness of $0.215 \mathrm{~mm}$ (from Eq. 10 (a)). As Fig. 11 shows, such a filter has a very small tuning rate (see Fig. 5), and even rotating the plate from $20^{\circ}$ to $60^{\circ}$ does not provide enough wavelength tuning. Moreover, as a result of the smaller tuning rate and narrower modulation bandwidth, the modulation depth of the filter varies significantly across the tuning range .

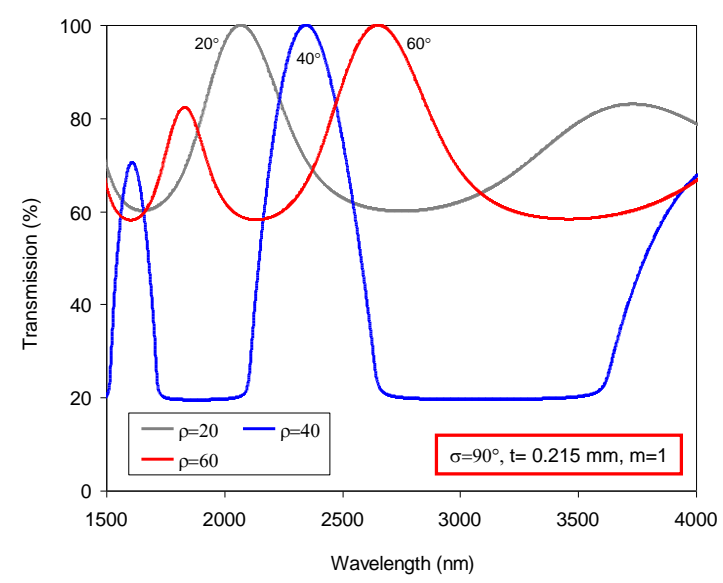

Fig. 11. Calculated transmission characteristics of a standing-wave Cr:ZnSe laser cavity around $2.35 \mu \mathrm{m}$, as a function of wavelength for different values of birefringent plates rotation angle (for $\rho$ in the range from $20^{\circ}$ to $60^{\circ}$ ). The calculation has been performed for a $0.215 \mathrm{~mm}$ thick magnesium fluoride birefringent plate with an optic axis orientation $(\sigma)$ of $90^{\circ}$. Plate rotation angles are chosen so that the laser tunes around the first order $(\mathrm{m}=1)$, where the FSR is largest.

\section{B. Broadband Filter Design for Ti:Sapphire Lasers}

Ti:Sapphire has a gain spectrum covering the region roughly from $\lambda_{1}=0.66 \mu \mathrm{m}$ to $\lambda_{2}=1.18 \mu \mathrm{m}$ [12], with a corresponding central wavelength of $0.85 \mu \mathrm{m}$ (harmonic mean). Then using Eq. (10.a), we find the optimum thickness for the birefringent filter as $2.2 \mathrm{~mm}$. Figure 12 shows the calculated transmission characteristics of a standingwave Ti:Sapphire laser containing a $2.2 \mathrm{~mm}$ thick magnesium fluoride BRF with an optic axis diving angle of $30^{\circ}$. We see that, as the filter angle is varied in the $10-18^{\circ}$ range, the transmission maxima of the filter shifts from $675 \mathrm{~nm}$ to $1250 \mathrm{~nm}$, and the modulation depth stays in the $50-60 \%$ range as the filter is tuned, which shows that this filter could successfully accomplish smooth tuning of Ti:Sapphire laser in its whole emission range. Note that the filters rejection at the stop band is weaker due to the lower refractive index of Ti:Sapphire compared to Cr:ZnSe (n $\mathrm{n}_{\text {sapphire}}=1.76$ q $_{\text {sapphire }}=0.86$ ).

Another point to note here is, in continuous wave laser operation, even though the FWHM of the resonance around $850 \mathrm{~nm}$ is quite large $(\sim 160 \mathrm{~nm})$, the optical line-width of the laser will be in the order of 100 s of picometers (same is true for the Cr:ZnSe laser discussed above). Note that the bandwidths we see here corresponds to a single round-trip filtering effect. During laser operation, the laser will observe this filter many times, and the effective filter bandwidth will be much narrower (depends on the cavity photon lifetime)[2]. Pairs of correctly designed etalons might further be inserted inside the cavity to narrow down the line width below $10 \mathrm{pm}$.

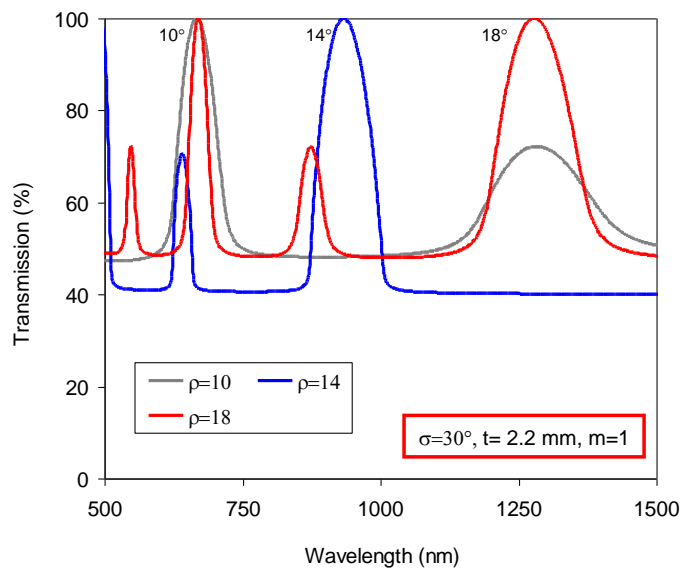

Fig. 12. Calculated transmission characteristics of a standing-wave Ti:Sapphire laser cavity around $0.85 \mu \mathrm{m}$, as a function of wavelength for different values of birefringent plates rotation angle (for $\rho$ in the range from $10^{\circ}$ to $18^{\circ}$ ). The calculation has been performed for a 2.2 $\mathrm{mm}$ thick magnesium fluoride birefringent plate with an optic axis orientation $(\sigma)$ of $30^{\circ}$. Plate rotation angles are chosen so that the laser tunes around the first order $(\mathrm{m}=1)$, where the FSR is largest.

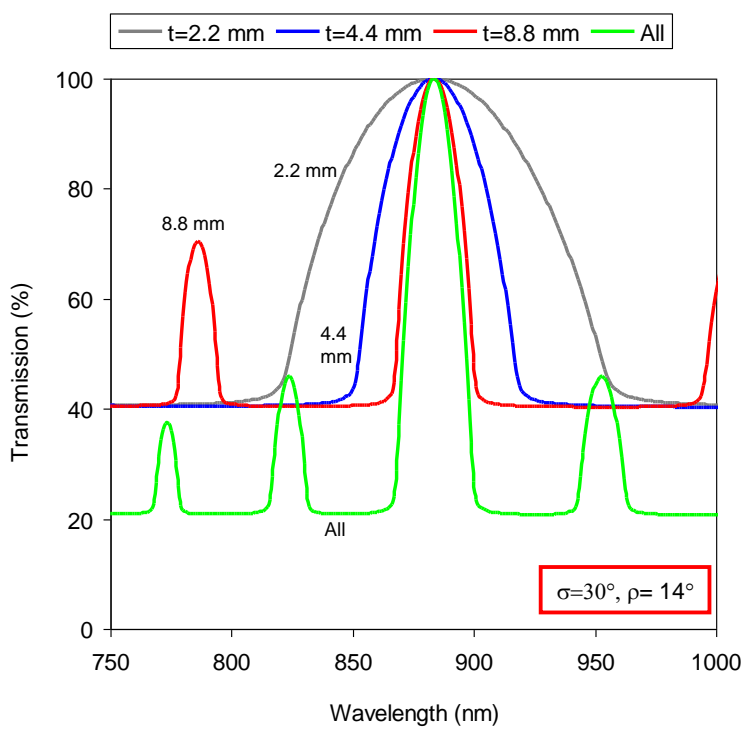

Fig. 13. Calculated transmission characteristics of a standing-wave Ti:Sapphire laser cavity around $0.85 \mu \mathrm{m}$ as a function of wavelength containing magnesium fluoride birefringent tuning plates with different thickness $(\mathrm{t}=2.2 \mathrm{~mm}, \mathrm{t}=4.4 \mathrm{~mm}$ and $\mathrm{t}=8.8 \mathrm{~mm})$. The calculation has been performed for an optic axis orientation $(\sigma)$ of $30^{\circ}$, and plate rotation angle $\rho$ of $14^{\circ}$. Calculated transmission results for a Ti:Sapphire cavity containing all the three birefringent filters simultaneously is also shown.

In mode-locked regime, with proper balance of self-phase modulation and dispersion, the large FWHM $(\sim 160 \mathrm{~nm})$ of the proposed filter should enable tuning of the Ti:Sapphire laser with sub50 -fs pulses. Tuning with longer (100-300 fs) pulses could also be achieved by proper setting of the total cavity dispersion [23]. If tuning with even longer pulses (picoseconds) are desired, one can add extra birefringent filters into the cavity to narrow down the filters FWHM (or operate the filter at an higher filter order (m) at the expense of narrower tuning range) [3]. For example, Fig. 13 shows the filtering characteristics of a Ti:Sapphire laser cavity containing three 
birefringent tuning plates all with an optic axis diving angle of $30^{\circ}$ and thickness of 2.2, 4.4 and $8.8 \mathrm{~mm}$. In this configuration, the filter bandwidth of the overall cavity (FWHM) reduces to around 25 nanometers. Note again that this bandwidth corresponds to a single round-trip filtering effect, and for the circulating intracavity pulse the effective filter bandwidth will be much narrower [2], and this configuration should ideally enable generation of tunable ps pulses.

\section{Broadband Filter Design for Other Laser Gain Media}

Above we have provided design examples for Cr:ZnSe and Ti:Sapphire lasers. In this section, we would like to provide Table 1, which lists optimum thickness values for magnesium fluoride birefringent filters for broadband tuning of several selected laser gain media. Note that, for gain media with similar emission wavelength range (such as Ti:Sapphire, Cr:LiSAF, Cr:LiCAF, and Alexandrite), a birefringent filter with the same thickness could be used. In preparing Table 1 , we have assumed a plate optic axis orientation $(\sigma)$ of $30^{\circ}$ for magnesium fluoride, which required the plate to be operated near its first order, at around a plate rotation angle $\rho$ of $13.6^{\circ}$. In general, Eq. 10 (a) behaves almost linearly and to first order, the optimum magnesium fluoride birefringent plate thickness for a laser gain media with a central wavelength of $\lambda_{c}$ could be estimated using $t_{\text {opt }}=2.93 \lambda_{c}-0.4$, where $\lambda_{c}$ is in micrometers and toptis in millimeters $\left(\sigma=30^{\circ}, \rho=13.6^{\circ}\right)$.

Table 1: Calculated values for optimum birefringent plate thickness for broadband tuning of selected laser gain media. Thickness optimization assumes a plate optic axis orientation $(\sigma)$ of $30^{\circ}$ for magnesium fluoride and $25^{\circ}$ for crystal quartz.

\begin{tabular}{|c|c|c|c|}
\hline Gain Medium & $\begin{array}{l}\text { Tuning Range } \\
\text { (nm) }\end{array}$ & $\begin{array}{l}\text { Optimum } \\
\mathrm{MgF}_{2} \text { filter } \\
\text { thickness } \\
(\mathrm{mm})\end{array}$ & $\begin{array}{l}\text { Optimum } \\
\text { quartz filter } \\
\text { thickness } \\
\text { (mm) }\end{array}$ \\
\hline Ce:LiCAF & $280-316[10,11]$ & \multirow{3}{*}{0.75} & \multirow{3}{*}{0.5} \\
\hline Ce:LiSAF & $288-313[24]$ & & \\
\hline Ce:LiLuF & $305-333$ [25] & & \\
\hline Ti:Sapphire & $660-1180[12]$ & \multirow{4}{*}{2.2} & \multirow{4}{*}{1.4} \\
\hline Alexandrite & $701-858[26,27]$ & & \\
\hline Cr:LiCAF & $720-885[28,29]$ & & \\
\hline Cr:LiSAF & $775-1042[22,30]$ & & \\
\hline Cr:Forsterite & $1130-1367$ [31] & 3.2 & 2 \\
\hline Cr:YAG & $1309-1596[32]$ & 3.7 & 2.4 \\
\hline Tm:YAG & $1870-2160[33]$ & \multirow{2}{*}{5.2} & \multirow{2}{*}{3.5} \\
\hline Tm:YLF & $1910-2070[34]$ & & \\
\hline Co: $\mathrm{MgF}_{2}$ & $1750-2500[35]$ & 5.4 & 4 \\
\hline Cr:ZnS & $1962-3195[14,36]$ & \multirow{3}{*}{6} & \multirow{3}{*}{ - } \\
\hline Cr:CdSe & $2180-3610$ [37-39] & & \\
\hline Cr:ZnSe & $1880-3349[13,14]$ & & \\
\hline Fe:ZnSe & $3900-4800$ [15] & \multirow{2}{*}{11.5} & \multirow[b]{2}{*}{-} \\
\hline Fe:ZnS & $3440-4190[40]$ & & \\
\hline Fe:CdSe & $4600-5900$ [41] & 18 & - \\
\hline
\end{tabular}

For the sake of completeness, in Table 1, we have also provided estimated optimum BRF thicknesses for crystal quartz birefringent tuning plates as well $\left(\sigma=25^{\circ}, \rho=23.55^{\circ}\right)$. For the case of crystal quartz, the optimum BRF thickness for a laser gain media with a central wavelength of $\lambda_{c}$ could be estimated using $t_{\text {opt }}=1.8 \lambda_{c}-0.1$, where $\lambda_{c}$ is in micrometers and $t_{\text {opt }}$ is in millimeters. As a reminder, the thickness values estimated in Table 1 is quite flexible, as it is already discussed with the simulations shown in Fig. 10.

\section{CONCLUSION}

In summary, we have investigated birefringent tuning plates made out of magnesium fluoride material as a tool in tuning of laser gain media with ultrabroad bandwidth. Our analysis has shown that, intracavity magnesium fluoride birefringent filters with an optic axis orientation $(\sigma)$ of $30^{\circ}$ could be efficiently used in smooth continuous-wave and femtosecond tuning of many solid-state gain media including Ti:Sapphire, Cr:ZnSe and Fe:ZnSe. A comparative optimization for crystal quartz indicates an optimum cut angle of $25^{\circ}$. We have further provided a simple formula in estimating the optimum plate thickness information in terms of the central emission wavelength of laser gain media. We hope that, the results presented in this paper will serve as a quick reference for researchers working in the are of broadly tunable lasers. Moreover, the general discussion on the effect of optic axis orientation on filter parameters should be useful for researchers in optimizing the filter specs for other applications as well.

Funding. (The Scientific and Technological Research Council of Turkey (TUBITAK, 114F191), European Union Marie Curie Career Integration Grant(PCIG11-GA-2012-321787).

Acknowledgment. We thank James G. Fujimoto of MIT for introducing us to the birefringent filter design problem.

\section{References}

1. S. M. Kobtsev and N. A. Sventsitskay, "Application of birefringent filters in continuous-wave tunable lasers: a review," Opt. Spectrosc. 73, 114-123 (1992).

2. S. Lovold, P. F. Moulton, D. K. Killinger, and N. Menwk, "Frequency Tuning Characteristics of a Q-Switched Co:MgF 2 Laser " IEEE J. Quantum Electron. QE-21, 202-208 (1985).

3. U. Demirbas, J. Wang, G. S. Petrich, S. Nabanja, J. R. Birge, L. A. Kolodziejski, F. X. Kartner, and J. G. Fujimoto, "100-nm tunable femtosecond Cr:LiSAF laser mode locked with a broadband saturable Bragg reflector," Appl. Opt. 56, 3812-3816 (2017).

4. A. L. Bloom, "Modes of a laser resonator containing tilted birefringent plates," J. Opt. Soc. Am. B 64, 447-452 (1974).

5. S. Zhu, "Birefringent filter with tilted optic axis for tuning dye lasers: theory and design," Appl. Opt. 29, 410-415 (1990).

6. K. Naganuma, G. Lenz, and E. P. Ippen, "Variable Bandwidth Birefringent Filter for Tunable Femtosecond Lasers," IEEE J. Quantum Electron. 28, 21422150 (1992).

7. C. H. Bair, "Birefringent filter design," (Google Patents, 1991).

8. G. H. Li and Y. Li, "Tuning Sensitivity of Dye-Laser Birefringent Filters," Appl. Opt. 29, 3462-3463 (1990).

9. U. Demirbas, R. Uecker, J. G. Fujimoto, and A. Leitenstorfer, "Multicolor lasers using birefringent filters: experimental demonstration with Cr:Nd:GSGG and Cr:LiSAF," Opt. Express 25, 2594-2607 (2017).

10. A. J. S. McGonigle, D. W. Coutts, and C. E. Webb, "530-mW 7-kHz cerium LiCAF laser pumped by the sum-frequency-mixed output of a copper-vapor laser," Opt. Lett. 24, 232-234 (1999).

11. B. Wellmann, O. Kitzler, D. J. Spence, and D. W. Coutts, "Linewidth narrowing of a tunable mode-locked pumped continuous-wave Ce:LiCAF laser," Opt. Lett. 40, 3065-3068 (2015).

12. P. F. Moulton, "Spectroscopic and laser characteristics of $\mathrm{Ti}_{2} \mathrm{Al}_{2} \mathrm{O}_{3}$," JOSA B 3 , 125-133 (1986).

13. U. Demirbas and A. Sennaroglu, "Intracavity-pumped Cr2+: ZnSe laser with ultrabroad tuning range between 1880 and 3100 nm," Opt. Lett. 31, 22932295 (2006).

14. E. Sorokin, I. T. Sorokina, M. S. Mirov, V. V. Fedorov, I. S. Moskalev, and S. B. Mirov, "Ultrabroad continuous-wave tuning of ceramic Cr:ZnSe and $\mathrm{Cr}: \mathrm{ZnS}$ lasers," in OSA / ASSP/LACSEA/LS\&C, (OSA, 2010).

15. J. Kernal, V. V. Fedorov, A. Gallian, S. B. Mirov, and V. V. Badikov, "3.9-4.8 $\mu \mathrm{m}$ gain-switched lasing of Fe:ZnSe at room temperature," Optics Express 13, 10608-10615 (2005).

16. M. J. Dodge, "Refractive Properties of Magnesium Fluoride," Appl. Opt. 23, 1980-1985 (1984).

17. D. R. Preuss and J. L. Gole, "Three-stage birefringent filter tuning smoothly over the visible region: theoretical treatment and experimental design," Appl. Opt. 19, 702-710 (1980).

18. X. Wang and J. Yao, "Transmitted and tuning characteristics of birefringent filters," Appl. Opt. 31, 4505-4508 (1992).

19. H. Wu, C. Zhang, and X. Bai, "A complete description of polarization and transmission of nonnormal incident rays in a uniaxial birefringent plate with arbitrary optic axis," Optics Communication 283, 4129-4134 (2010). 
20. T. Yerebakan, U. Demirbas, S. Eggert, R. Bertram, P. Reiche, and A. Leitenstorfer, "Red diode pumped Cr:Nd:GSGG laser: two-color modelocked operation," J. Opt. Soc. Am. B 34, 1023-1032 (2017).

21. C. G. Trevino-Palacios, O. J. Zapata-Nava, E. V. Mejia-Uriarte, N. Qureshi, G. Paz-Martinez, and O. Kolokolstev, "Dual wavelength continuous wave laser using a birefringent filter," Journal of the European Optical Society - Rapid publications 8, 13021 (2013).

22. U. Demirbas and I. Baali, "Power and efficiency scaling of diode pumped $\mathrm{Cr}$ : LiSAF lasers: $770-1110 \mathrm{~nm}$ tuning range and frequency doubling to 387-463 nm," Opt. Lett. 40, 4615-4618 (2015).

23. U. Demirbas, G. S. Petrich, D. Li, A. Sennaroglu, L. A. Kolodziejski, F. X. Kärtner, and J. G. Fujimoto, "Femtosecond tuning of Cr:Colquiriite lasers with AlGaAs-based saturable Bragg reflectors," JOSA B 28, 986-993 (2011).

24. J. F. Pinto, L. Esterowitz, and G. J. Quarles, "High performance Ce :LiSrAIF /LiCaAIF lasers with extended tunability," Electron. Lett. 31, 2009-2010 (1995).

25. A. J. S. McGonigle, S. Girard, D. W. Coutts, and R. Moncorgé, "10-kHz continuously tunable Ce:LiLuF laser," Electron. Lett. 35, 1640-1641 (1999).

26. J. C. Walling, O. G. Peterson, H. P. Jenssen, R. C. Morris, and E. W. O'Dell, "Tunable alexandrite lasers," IEE Journal of Quantum Electronics 16, 13021315 (1980)

27. J. W. Kuper, T. Chin, and H. E. Aschoff, "Extended tuning of Alexandrite laser at elevated temperetures," in Advanced Solid State Lasers, (OSA, Salt Lake City, Utah, 1990).

28. S. A. Payne, L. L. Chase, H. W. Newkirk, L. K. Smith, and W. F. Krupke, "LiCaAlF $6: \mathrm{Cr}^{3+}$ a promising new solid-state laser material," IEEE J. Quantum Electron. 24, 2243-2252 (1988).

29. U. Demirbas, I. Baali, D. A. E. Acar, and A. Leitenstorfer, "Diode-pumped continuous-wave and femtosecond Cr:LiCAF lasers with high average power in the near infrared, visible and near ultraviolet," Opt. Express 23, 8901-8909 (2015).

30. M. Stalder, B. H. T. Chai, and M. Bass, "Flashlamp pumped Cr:LiSrAlF 6 laser," Appl. Phys. Lett. 58, 216-218 (1991).

31. V. G. Baryshevskii, M. V. Korzhik, A. E. Kimaev, M. G. Livshits, V. B. Pavlenko, M. L. Meil'man, and B. I. Minkov, "Tunable chromium forsterite laser in the near IR region," Journal of Applied Spectroscopy (USSR) 53, 675-676 (1990).

32. S. Kuck, K. Petermann, U. Pohlmann, U. Schonhoff, and G. Huber, "Tunable room-temperature laser action of a Cr-4+-doped Y3ScxAl5-xO12," Applied Physics B 58, 153-156 (1994).

33. R. C. Stoneman and L. Esterowitz, "Efficient, broadly tunable, laser-pumped Tm:YAG and Tm:YSGG cw lasers," Opt. Lett. 15, 486-488 (1990).

34. A. Dergachev, K. Wall, and P. F. Moulton, "A CW Side-Pumped Tm:YLF Laser," in OSA TOPS Advanced Solid State Lasers, (OSA, 2002), 343-346.

35. D. Welford and P. F. Moulton, "Romm-temperature operation of a Co:MgF laser," Opt. Lett. 13, 975-977 (1988).

36. I. T. Sorokina, E. Sorokin, S. Mirov, V. Fedorov, V. Badikov, V. Panyutin, and K. I.Schaffers, "Broadly tunable compact continuous-wave $\mathrm{Cr}^{2+}: \mathrm{ZnS}$ laser," Opt. Lett. 27, 1040-1042 (2002).

37. J. McKay, K. L. Schepler, and G. C.Catella, "Efficient grating-tuned midinfrared $\mathrm{Cr}^{2+}$ :CdSe laser," Opt. Lett. 24, 1575-1577 (1999).

38. V. A. Akimov, M. P. Frolov, Y. V. Korostelin, V. I. Kozlovsky, A. I. Landman, Y. P. Podmar'kov, Y. K. Skasyrsky, and A. A. Voronov, "Pulsed broadly tunable room-temperature Cr2+:CdS laser," Appl. Phys. B 97, 793-797 (2009).

39. V. A. Akimov, V. I. Kozovskii, Y. V. Korostelin, A. I. Landman, Y. P. Podmar'kov, Y. K. Skasyrskii, and M. P. Frolov, "Efficient pulsed Cr2+: CdSe laser continuously tunable in the spectral range from 2.26 to 3.61 ," Quantum Electronics 38, 205-208 (2008).

40. M. P. Frolov, Y. V. Korostelin, V. I. Kozlovsky, Y. P. Podmar'kov, S. A. Savinova and Y. K. Skasyrsky, "3 J pulsed Fe:ZnS laser tunable from 3.44 to $4.19 \mathrm{mu}$ m," Laser Physics Letters 12(2015).

41. V. I. Kozlovsky, V. A. Akimov, M. P. Frolov, Y. V. Korostelin, A. I. Landman, V. P. Martovitsky, V. V. Mislavskii, Y. P. Podmar'kov, Y. K. Skasyrsky, and A. A. Voronov, "Room-temperature tunable midinfrared lasers on transitionmetal doped II-VI compound crystals grown from vapor phase," Physica Status Solidi B-Basic Solid State Physics 247, 1553-1556 (2010). 